\title{
Spatial propagation of coherency matrix in polarization optics
}

\author{
Vincent Devlaminck \\ LAGIS - UMR CNRS 8219 \\ Université Lille 1, Sciences et Technologies - 59655 France \\ e.mail : vincent.devlaminck@univ-lille1.fr
}

\section{1 - Introduction}

In his effort to describe the state of the light at every point $z$ in an optical system along the light path, Jones introduced the notion of $N$ matrices based on a layered-medium interpretation [1]. The $\mathrm{N}$ matrix at the point $z$, where $z$ is measured along the light path is defined by:

$$
\mathbf{N}=\frac{\mathrm{d} \mathbf{J}}{\mathrm{d} z} \mathbf{J}^{-1}
$$

where $\mathbf{J}$ is the Jones matrix of the optical element at the point $z$. Later, Azzam extended this approach [2] to the partially polarized light propagating through non depolarizing media and found that the Stokes vector of the light obeys the equation:

$$
\frac{\mathrm{d} \mathbf{S}}{\mathrm{d} z}=\mathbf{m S}
$$

where $\mathbf{m}$ is termed the differential Mueller matrix and $\mathbf{S}$ stands for the Stokes vector of the light at distance $z$ into the medium. Azzam also derived the relations between the entries of $\mathbf{N}$ and $\mathbf{m}$ differential matrices for non depolarizing media. However, the formal relation between these both matrices was formulated by Barakat [3]. From the concept of exponential versions of the MuellerJones matrices and properties of the Kronecker product $\otimes$ of matrices, Barakat established the following relation:

$$
\mathbf{m}=\boldsymbol{\Lambda}^{\dagger}\left(\mathbf{N} \otimes \mathbf{I}+\mathbf{I} \otimes \mathbf{N}^{\dagger}\right) \boldsymbol{\Lambda}
$$

where $\uparrow$ and $*$ stand for a Hermitian and complex conjugate respectively, $\mathbf{I}$ is the identity matrix of rank 2 and:

$$
\boldsymbol{\Lambda}=\frac{\mathbf{1}}{\sqrt{\mathbf{2}}}\left[\begin{array}{cccc}
1 & 1 & 0 & 0 \\
0 & 0 & 1 & -\mathrm{i} \\
0 & 0 & 1 & \mathrm{i} \\
1 & -1 & 0 & 0
\end{array}\right]
$$

If $A$ is an $m$-by- $n$ matrix and $B$ is a $p$-by- $q$ matrix, then the Kronecker product is the $m p$-by- $n q$ block matrix defined by:

$$
\mathbf{A} \otimes \mathbf{B}=\left[\begin{array}{ccc}
\mathrm{a}_{11} \mathbf{B} & \cdots & \mathrm{a}_{1 \mathrm{n}} \mathbf{B} \\
\vdots & \ddots & \vdots \\
\mathrm{a}_{\mathrm{m} 1} \mathbf{B} & \cdots & \mathrm{a}_{\mathrm{mn}} \mathbf{B}
\end{array}\right]
$$

The basic point that I wish to stress is that the state of light beam or the path in a medium to go from a physical situation at distance $z_{1}$ to another one at distance $z_{2}$ should be related to the correlation between both these physical situations. This point will be developed in the following but using coherency matrices concept rather than Stokes-Mueller matrices approach. 


\section{2 - Coherency matrix as random process.}

First, we do a reminder of definitions and relations between coherency matrices associated to the components of the beam of light. These elements are considered as random variables which can be described by their covariance matrix. Generalization of these random variables to functions of space is addressed using the concept of random process.

\section{1 - Light beam formalism}

The coherency matrix $\boldsymbol{\Phi}$ is the covariance matrix of the components of the $2 \mathrm{D}$ complex electric field vector E. Such a matrix is given by Eq. (6) :

$$
\boldsymbol{\Phi}=\left\langle\mathbf{E}(k) \mathbf{E}^{\dagger}(k)\right\rangle_{k}
$$

where the symbol \langle\rangle$_{\mathrm{k}}$ denotes ensemble averaging over the elementary events $k$. As a covariance matrix, $\boldsymbol{\Phi}$ is a Hermitian nonnegative definite matrix. So, its eigenvalues are real and positive. As a $2 \times 2$ complex matrix, $\boldsymbol{\Phi}$ can be expanded as a linear combination of the four Pauli matrices $\boldsymbol{\sigma}_{\mathrm{i}}$ as given by Eq. (7):

$$
\boldsymbol{\Phi}=\sum_{\mathrm{j}=0}^{3} \mathrm{~S}_{\mathrm{j}} \boldsymbol{\sigma}_{\mathrm{j}}
$$

where $S=\left[\begin{array}{llll}S_{0} & S_{1} & S_{2} & S_{3}\end{array}\right]^{T}$ stands for the Stokes vector associated with the light beam. Another complete set of four matrices could be used for the decomposition but the Pauli matrices are the natural ones to use because they underlie the geometry of the Poincaré sphere [4] .

\subsection{Coherency as function of space}

The relevant quantities at distance $\mathrm{z}$ into a medium or for a light beam can be depicted by a covariance $\mathbf{H}(z)$ with $\mathbf{H}(z)=\boldsymbol{\Phi}(z)$.

Generalization of these previous definitions to functions of space, leads to the concept of random process [5]. In the following, $\mathbf{E}(k, z)$ will represent the complex-valued Jones vector with independent variable $z$ to each elementary event $k$. A second order probability density function $\mathrm{f}$ may be associated to this process and the corresponding second order joint moment or classically termed "statistical correlation" between the two random vectors $\mathbf{E}\left(k, z_{1}\right)$ and $\mathbf{E}\left(k, z_{2}\right)$ is defined as :

$$
\mathbf{G}\left(z_{1}, z_{2}\right)=\left\langle\mathbf{E}\left(k, z_{2}\right) \mathbf{E}^{\dagger}\left(k, z_{1}\right)\right\rangle_{k}=\int_{\mathrm{K}} \mathbf{E}\left(k, z_{2}\right) \mathbf{E}^{\dagger}\left(k, z_{1}\right) \mathrm{f}\left(k, z_{1}, z_{2}\right) \mathrm{d} k
$$

Where $\mathrm{K}$ stands for the set of all possible events.

$\mathbf{H}\left(z_{1}\right)=\mathbf{G}\left(z_{1}, z_{1}\right)$ and $\mathbf{H}\left(z_{2}\right)=\mathbf{G}\left(z_{2}, z_{2}\right)$ is obvious with these definitions.

Introducing the covariance matrix of vector $\boldsymbol{\Omega}^{\mathrm{T}}=\left[\mathbf{E}\left(k, z_{1}\right)^{\mathrm{T}} \quad \mathbf{E}\left(k, z_{2}\right)^{\mathrm{T}}\right]$, is a way to obtain a complete second order statistical characteristics of this random process at two points $z_{1}$ and $z_{2}$. This matrix will be denoted by $\boldsymbol{\Sigma}$ this matrix, with:

$$
\boldsymbol{\Sigma}=\left\langle\boldsymbol{\Omega}\left(k, z_{1}, z_{2}\right) \mathbf{\Omega}^{\dagger}\left(k, z_{1}, z_{2}\right)\right\rangle_{k}=\left[\begin{array}{cc}
\mathbf{H}\left(z_{1}\right) & \mathbf{G}^{\dagger}\left(z_{1}, z_{2}\right) \\
\mathbf{G}\left(z_{1}, z_{2}\right) & \mathbf{H}\left(z_{2}\right)
\end{array}\right]
$$

Though $\boldsymbol{\Sigma}$ as a covariance matrix, is a Hermitian positive semi definite matrix, we restrict our approach to the elements of $H P D(4)$ the manifold of Hermitian Positive Definite matrices of dimension 4 (their eigenvalues must be strictly positive). With this hypothesis, $\boldsymbol{\Sigma}$ is non singular. In the same way, $\mathbf{H}\left(z_{1}\right)$ and $\mathbf{H}\left(z_{2}\right)$ are restricted to belong to $H P D(2)$. It is clear that we do not take into 
account the completely polarized light. In polarization optics, these properties for the beam of light are depicted using the notion of polarization degree.

Defining a path between $\mathbf{H}\left(z_{1}\right)$ and $\mathbf{H}\left(z_{2}\right)$ according to a given expression of $\mathbf{G}\left(z_{1}, z_{2}\right)$ will be done below.

\section{Spatial interpolation of coherency matrices.}

The main idea we develop is related to an equivalent approach proposed by Réfrégier [6] in order to analyse symmetries in coherence theory and intrinsic degrees of coherence of light.

\subsection{Equivalent physical situations}

If the random vector $\mathbf{E}(k, z)$ at two points $z_{1}$ and $z_{2}$ is multiplied by non-singular deterministic matrices, the intrinsic randomness properties should not be modified since reversible and deterministic linear transformations do not affect the amount of randomness between the two vectors (for more details, see [6] and [7]).

With this remark, two physical situations (depicted by their associated matrices $\boldsymbol{\Sigma}_{1}$ and $\boldsymbol{\Sigma}_{2}$ ) related by such a transformation can be considered as equivalent (from a second order statistical point of view) and will be noted by $\Sigma_{1} \equiv \Sigma_{2}$. More precisely:

$$
\boldsymbol{\Sigma}_{\mathbf{1}}\left(z_{1}, z_{2}\right) \equiv \boldsymbol{\Sigma}_{\mathbf{2}}\left(z_{1}, z_{2}\right) \Leftrightarrow \exists \boldsymbol{\Gamma}=\left[\begin{array}{cc}
\boldsymbol{\Pi}_{1} & 0 \\
0 & \boldsymbol{\Pi}_{2}
\end{array}\right] \in G L(2, \mathbb{C}) \otimes G L(2, \mathbb{C}) / \boldsymbol{\Sigma}_{\mathbf{2}}=\boldsymbol{\Gamma} \boldsymbol{\Sigma}_{\mathbf{1}} \boldsymbol{\Gamma}^{\dagger}=\varphi\left(\boldsymbol{\Gamma}, \boldsymbol{\Sigma}_{\mathbf{1}}\right)
$$

where $G L(2, \mathbb{C})$ is the group of non singular $2 \times 2$ matrices with complex entries and $L G=G L(2, \mathbb{C}) \otimes G L(2, \mathbb{C})$ is the tensorial product of $G L(2, \mathbb{C})$ with itself. Modifying the correlation properties by the transformation action is avoided if the $\Gamma$ matrices are block diagonal. From a mathematical point of view, Eq. (10) defines a group action $\varphi$ of the Lie group $L G$ on the manifold $H P D(4)$ as a mapping $\varphi: L G \times H P D(4) \rightarrow H P D(4)$ satisfying two conditions ( [8], p.90):

$$
\begin{aligned}
& \forall x \in H D P(4), \varphi(e, x)=x \\
& \forall\left(g_{1}, g_{2}\right) \in L G \times L G, \forall x \in H D P(4), \varphi\left(g_{1}, \varphi\left(g_{2}, x\right)\right)=\varphi\left(g_{1} g_{2}, x\right)
\end{aligned}
$$

where $e$ is the identity element of $L G$. Such an action is termed transitive on a sub-manifold $M$ of $H P D(4)$ if for every pair $p$ and $q$ of $M$ there is a $g \in L G$ such that $\varphi(g, p)=q$. This means that any two points of this sub-manifold are alike under the G-action. Since $L G$ is reduced to block diagonal matrices it is obvious that $\varphi$ is not a transitive action on $\operatorname{HPD}(4)$. But for a given element $\boldsymbol{\Sigma}_{0}$ of $H P D(4)$ is possible to find out an associated sub-manifold where $\varphi$ acts transitively. This submanifold $O$ is termed the orbit of $\Sigma_{0}$ defined as:

$$
O\left(\boldsymbol{\Sigma}_{0}\right)=\left\{\boldsymbol{\Sigma}=\boldsymbol{\Gamma} \boldsymbol{\Sigma}_{0} \boldsymbol{\Gamma}^{\dagger}, \forall \boldsymbol{\Gamma} \in L G\right\}
$$

As example for $\boldsymbol{\Sigma}_{0}=\mathbf{I d}$, the identity matrix of dimension $4, O(\mathbf{I d})$ is $H P D(2) \otimes H P D(2)$ as the set of $\Sigma=\Gamma \Gamma$ for all the possible elements $\Gamma$ belonging to $L G$.

It is obvious that two elements in the same orbit verify the equivalence relation defined by Eq. (10). These orbits are in fact the equivalence classes for the group action acting on the manifold. Thus, an orbit $O(\boldsymbol{\Sigma})$ can be associated to every element $\boldsymbol{\Sigma}$ (defined by related elements $\mathbf{H}\left(z_{1}\right), \mathbf{H}\left(z_{2}\right)$ and $\mathbf{G}\left(z_{1}, z_{2}\right)$, and the group action $\varphi$ acts transitively on this sub-manifold. 


\subsection{Geodesic curve as path between physical situations}

The main reason to only consider the action in a special case, transitive action, is related to the circumstances one can be sure that a manifold can be endowed with a Riemannian metric relative to which the transformations defined by the group action are isometries. In order to have a positive answer to this question, $\varphi$ have to act transitively on this manifold. Others mathematical properties must be verified but are out of the scope of this paper and the reader is referred to [8] and [9] for the definitions and demonstrations of similar properties.

By this way, distance, geodesics and the length of curves can be defined on each of the submanifolds $O(\boldsymbol{\Sigma})$. Let $p$ be a point of $O(\boldsymbol{\Sigma})$, and $X_{p}$ a tangent vector at $p$, there is a unique geodesic $\gamma_{(p, X)}$ $(z)$ on $O(\boldsymbol{\Sigma})$ with initial point $\gamma_{(p, X)}(0)=p$ and tangent vector $\gamma_{(p, X)}^{\prime}(0)=X_{p} \cdot \gamma_{(p, X)}(1)$, the point $x$ on the geodesic at which the parameter takes the value 1, is the exponential mapping noted $\operatorname{Exp}_{p}(X)$. The inverse mapping of Exponential mapping is defined by $\quad X=\log _{p}(x)$ and named Log mapping. With these notations we have [8]:

$$
\gamma_{(p, X)}(z)=\operatorname{Exp}_{p}\left[z \log _{p}(x)\right] \text { with } x=\operatorname{Exp}_{p}(X)
$$

Thus $\gamma_{(p, X)}(z)$ can be considered as an interpolation curve from $p$ to $x$ according to Eq. (13).

The major point that I wish to stress is that the geodesic curve between $p$ and $x$ is an optimal path (as the curve with the shortest length between any two points- the reader is referred to Appendix A for the definitions of the associated Riemannian metric and to section 2 of [9] where the definition of the length of curves on this Riemannian space and related notions are recalled) fully included in $O(\boldsymbol{\Sigma})$. Thus every point of $\gamma_{(p, X)}(z)$ for $0 \leq z \leq 1$ is element of the orbit of $\boldsymbol{\Sigma}$ and the physical situations can be considered as equivalent from a second order statistical point of view as defined by Eq. (10). Last but not least, as $\varphi$ acts transitively on this manifold and is an isometry.

Let us now consider a point $x$ of $H P D(4)$ :

$$
x=\left[\begin{array}{cc}
\mathbf{H}_{1} & \mathbf{G}^{\dagger} \\
\mathbf{G} & \mathbf{H}_{2}
\end{array}\right]
$$

corresponding to a physical situation $\boldsymbol{\Sigma}$ as described by Eq. (9) where $\mathbf{H}_{\mathrm{i}}$ and $\mathbf{G}$ stand for $\mathbf{H}\left(z_{\mathrm{i}}\right)$ and $\mathbf{G}\left(z_{1}, z_{2}\right)$ respectively. A point $p$ defined by:

$$
p=\left[\begin{array}{cc}
\mathbf{H}_{1} & \left(\mathbf{H}_{1}^{1 / 2} \mathbf{H}_{2}^{-1 / 2} \mathbf{G}\right)^{\dagger} \\
\mathbf{H}_{1}^{1 / 2} \mathbf{H}_{2}^{-1 / 2} \mathbf{G} & \mathbf{H}_{1}
\end{array}\right]
$$

is an element of the orbit $O(\boldsymbol{\Sigma})$ since :

$$
g x g^{\dagger}=p \text { for } g=\left[\begin{array}{cc}
\mathbf{I d} & \mathbf{0} \\
\mathbf{0} & \mathbf{H}_{1}^{1 / 2} \mathbf{H}_{2}^{-1 / 2}
\end{array}\right]
$$

Thus $\gamma_{(p, X)}(z)$ given by Eq. (13) with $p$ and $x$ given by Eq. (14-15) defines a path from $p$ to $x$ without any change of the physical situation since every point belonging to the curve can be considered as equivalent, at least from a second order statistical point of view. Restricting $\gamma_{(p, X)}(z)$ to the sub-matrix $\gamma_{\mathrm{sm}(p, X)}(z)$ built from the extraction of the down right block of $\gamma_{(p, X)}(z)$ gives eventually the equation of the path from $\mathbf{H}_{1}=\gamma_{\mathrm{sm}(p, X)}(0)$ to $\mathbf{H}_{2}=\gamma_{\mathrm{sm}(p, X)}(1)$.

In general, we do not have a straightforward expression of this path since further study of the geodesic curves on $O(\boldsymbol{\Sigma})$ needs more detailed computations using local coordinates. Nevertheless there is at least one case where an explicit solution can be found out without any reference to a local 
coordinates frame.

Let us consider the case where the random vectors at points $z_{1}$ and $z_{2}$ are uncorrelated. Under the hypothesis of null mean value, we have $\mathbf{G}=\mathbf{0}$ in Eq. (14). It is quite obvious from Eq. (12) that we are now considering $O(\mathbf{I d})$, it means $H P D(2) \otimes H P D(2)$ as previously mentioned.

As $O(\mathbf{I d}) \subset G L$ if $p \in O(\mathbf{I d})$ ( $p$ is block diagonal) we can define $\mathrm{g} \in G L$ as :

$$
g=p^{1 / 2}=\left[\begin{array}{cc}
\mathbf{H}_{1}^{1 / 2} & \mathbf{0} \\
\mathbf{0} & \mathbf{H}_{2}^{1 / 2}
\end{array}\right]
$$

Since it is well known (see [10] for instance) that the exponential mapping at the matrix identity point is the classical matrix exponential we have $\gamma_{(\text {Id } Y)}(z)=\exp (z Y)$ where $Y$ stands for a tangent vector at identity. As the identity matrix can be brought to any matrix $p$ by the group action choosing $g=p^{1 / 2}$, and this action is an isometry, we have:

$$
\gamma_{(p, X)}(z)=\varphi\left[p^{1 / 2}, \gamma_{(I d, Y)}(z)\right]=p^{1 / 2} \exp (z Y)\left(p^{1 / 2}\right)^{\dagger}
$$

Likewise, the tangent vector $X$ at $p$ can be transported to the tangent vector $Y$ at identity by the group action choosing $g=p^{-1 / 2}$. And we can write

$$
Y=d \varphi\left(p^{-1 / 2}, X\right)=p^{-1 / 2} X\left(p^{-1 / 2}\right)^{\dagger} \text { with } X=\log _{p}(x)
$$

After substituting $Y$ and $X$, we finally have the following explicit expression of the geodesic curve:

$$
\begin{aligned}
\gamma_{(p, X)}(z) & =\operatorname{Exp}_{p}(z Y) \\
& =p^{1 / 2} \exp \left[z p^{-1 / 2} X\left(p^{-1 / 2}\right)^{\dagger}\right]\left(p^{1 / 2}\right)^{\dagger} \\
& =p^{1 / 2} \exp \left[z \log \left[p^{-1 / 2} x\left(p^{-1 / 2}\right)^{\dagger}\right]\right]\left(p^{1 / 2}\right)^{\dagger}
\end{aligned}
$$

This last equation gives for $p$ and $x$ in $O(\mathbf{I d})$ defined by:

$$
p=\left[\begin{array}{cc}
\mathbf{H}_{1} & \mathbf{0} \\
\mathbf{0} & \mathbf{H}_{1}
\end{array}\right] \text { and } x=\left[\begin{array}{cc}
\mathbf{H}_{1} & \mathbf{0} \\
\mathbf{0} & \mathbf{H}_{2}
\end{array}\right]
$$

the following equations:

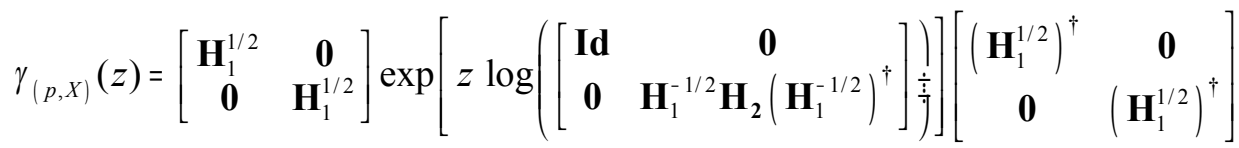

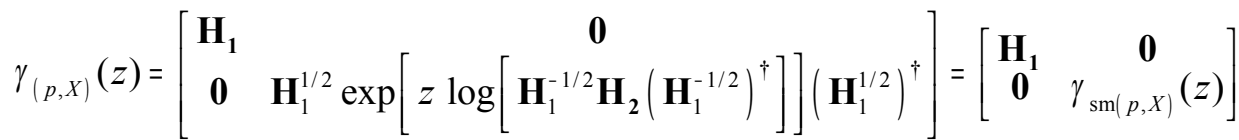

Restricting $\gamma_{(p, X)}(z)$ to its down right block gives eventually the equation of the path from $=\gamma_{\mathrm{sm}(p, X)}(0)$ to $\mathbf{H}_{2}=\gamma_{\mathrm{sm}(p, X)}(1)$.

It is worth noticing that the expression we derived in Eq. (22) is exactly the same as we already established in a previous paper [9]. But the second order statistical properties (un-correlation hypothesis) underlying this solution are now clearly established. Other second order statistical characteristics of this random process lead to define the path from Eq. (13) with $p$ and $x$ given by Eq. (14-15). 


\section{Physical significance and properties of interpolation process}

We address now the question of the physical significance of this interpolation process. Since the interpolation curve between $\mathbf{H}_{1}$ and $\mathbf{H}_{2}$ may also be defined by the knowledge of the initial point $\mathbf{H}_{1}$ and its tangent vector at this point, we will adopt the following notation $\gamma_{\mathrm{sm}(p, X)}(z)=\mathbf{H}_{(H 1, W)}(z)$ simply meaning that $\mathbf{H}_{(H I, W)}(z)$ is the interpolation curve going through $\mathbf{H}_{1}$ with tangent vector $\mathbf{W}$ such that $\mathbf{H}_{(H 1, W)}(1)=\mathbf{H}_{2}$. It is straightforward to see that $\mathbf{W}=(\mathrm{d} \mathbf{H} / \mathrm{d} z)_{z=0}$ is given by:

$$
\mathbf{W}=\mathbf{H}_{1}^{1 / 2} \log \left[\mathbf{H}_{1}^{-1 / 2} \mathbf{H}_{2}\left(\mathbf{H}_{1}^{-1 / 2}\right)^{\dagger}\right]\left(\mathbf{H}_{1}^{1 / 2}\right)^{\dagger}
$$

From general results of differentiable geometry for the invariant metrics on affine symmetric space [10], the geodesics are generated by the action of the one parameter subgroups of the acting Lie group. Since we are dealing with the Lie group $L G=G L(2, \mathbb{C}) \otimes G L(2, \mathbb{C})$ acting on $O(\mathbf{I d})$, the geodesics $\left.\mathbf{H}_{(H I}, W\right)(z)$ are generated by the action of classical matrix exponentials $\exp (\mathrm{zN})$ as well known one parameter subgroups of $G L(2, \mathbb{C})$. Thus we have:

$$
\mathbf{H}_{(H l, W)}(z)=\exp (z \mathbf{N}) \mathbf{H}_{\mathbf{1}}[\exp (z \mathbf{N})]^{\dagger} \text { with } \mathbf{W}=\mathbf{N H}_{\mathbf{1}}+\mathbf{H}_{\mathbf{1}} \mathbf{N}^{\dagger}
$$

where the condition on $\mathbf{W}$ is known as the Sylvester equation. This condition is necessary since $\mathbf{W}=(\mathrm{d} \mathbf{H} / \mathrm{d} z)_{z=0}$. For hermitian matrices, an explicit solution of this equation is given by [11] :

$$
\mathbf{N}=\frac{1}{2}\left[\mathbf{W} \mathbf{H}_{1}^{-1}+\mathbf{H}_{1}^{1 / 2} \mathbf{F}\left(\mathbf{H}_{1}^{-1 / 2}\right)^{\dagger}\right]
$$

where $\mathbf{F}$ is free skew-hermitian matrix.

The congruence relation of Eq. (24) corresponds to the linear transformation of the electric field by a Jones matrix $\mathbf{J}(z)=\exp (z \mathbf{N})$. $\mathbf{H}_{1}$ is the coherency matrix of the incident beam and $\left.\mathbf{H}_{(H I}, W\right)(z)$ is the coherency matrix of the beam at distance $z$ into the medium with $\left.\mathbf{H}_{(H I}, w\right)(1)=\mathbf{H}_{2}$. The associated Jones matrix $\mathbf{J}(z)=\exp (z \mathbf{N})$ is the expression of the Jones matrix of a uniform medium as introduced by Jones in the seventh paper of his series [1]. The statement that the medium is uniform means that $\mathbf{N}$ is independent of $z$ (Jones used the term homogeneous, nevertheless we will use uniform since the homogeneous property is usually reserved for a material having a Jones matrix with two orthogonal eigenpolarizations [4]).

The physical meaning of the interpolation technique based on the affine invariant distance is thus obvious. Interpolating coherency matrix from $\mathbf{H}_{1}$ to $\mathbf{H}_{2}$ describes the propagation of a light beam into a uniform medium (characterized by its $N$-matrix) in such a way that the emerging light at distance $\mathrm{z}=1$ has a coherency matrix $\mathbf{H}_{2}$. It is worth noticing that the $\mathbf{N}$ matrix is defined by $\mathbf{H}_{1}$ and $\mathbf{H}_{2}$ and $\mathbf{F}$ since from Eq. (25) and Eq. (23):

$$
\mathbf{N}=\frac{1}{2} \mathbf{H}_{1}^{1 / 2}\left[\log \left[\mathbf{H}_{1}^{-1 / 2} \mathbf{H}_{2}\left(\mathbf{H}_{1}^{-1 / 2}\right)^{\dagger}\right]+\mathbf{F}\right]\left(\mathbf{H}_{1}^{-1 / 2}\right)^{\dagger}
$$

However Eq. (25) is a necessary condition but not a sufficient one for $\mathbf{N}$ verifying $\mathbf{H}_{(H 1, w)}(1)=\mathbf{H}_{2}$. The skew-hermitian matrix $\mathbf{F}$ must verify (see Appendix B) the following commutation relation:

$$
\left[\log \left[\mathbf{H}_{1}^{-1 / 2} \mathbf{H}_{2}\left(\mathbf{H}_{1}^{-1 / 2}\right)^{\dagger}\right], \mathbf{F}\right]=0
$$

(where $[\mathbf{A}, \mathbf{B}]=\mathbf{A B}-\mathbf{B A})$ in order to cover the same path as the geodesic $\mathbf{H}_{(H 1, W)}(z)$ defined by $\quad$ Eq. ( 
22).

For an incident beam defined by a coherency matrix $\mathbf{H}_{1}$, if $\mathbf{H}_{2}$ is the coherency matrix of the emerging light beam, the Jones matrix of the uniform medium at distance $\mathrm{z}$ into the medium, is $\mathbf{J}(z)=\exp (z \mathbf{N})$ with $\mathbf{N}$ given by Eq. (26). Obtaining a more explicit expression for $\mathbf{J}$, is straightforward:

$$
\mathbf{J}(z)=\mathbf{H}_{1}^{1 / 2} \exp \left[\frac{z}{2} \log \left[\mathbf{H}_{1}^{-1 / 2} \mathbf{H}_{2}\left(\mathbf{H}_{1}^{-1 / 2}\right)^{\dagger}\right]\right] \cdot \exp \left[\frac{z}{2} \mathbf{F}\right]\left(\mathbf{H}_{1}^{-1 / 2}\right)
$$

It is thus possible to identify the Jones matrix of this uniform medium up to a unitary matrix.

Let us first consider the example of an unpolarized incident light beam with a coherency matrix $\mathbf{H}_{\mathbf{1}}=\mathbf{I d}$ and an emerging light with a coherency matrix $\mathbf{H}_{2}=$ Id. From Eq. (28), we have:

$$
\mathbf{J}(z)=\exp \left[\frac{z}{2} \mathbf{F}\right]
$$

where $\mathbf{F}$ is any skew-hermitian matrix since $\mathbf{F}$ must be a skew-hermitian matrix that commutes with null matrix. $\mathbf{J}$ is thus any unitary matrix. This is obviously the only way to keep an unpolarized light when a Jones matrix is acting on a unpolarized light.

For an unpolarized incident light beam and an emerging light with a coherency matrix $\mathbf{H}_{2}, \mathbf{J}$ has the following expression:

$$
\mathbf{J}(z)=\exp \left[\frac{z}{2} \log \left(\mathbf{H}_{2}\right)\right] \cdot \exp \left[\frac{z}{2} \mathbf{F}\right]
$$

$\mathbf{J}$ is the product of a hermitian matrix and a unitary one. Since both these matrices commute, $\mathbf{J}$ is a normal matrix $\left(\mathbf{J J}^{\dagger}=\mathbf{J}^{\dagger} \mathbf{J}\right)$ and the corresponding optical element has two orthogonal eigenpolarizations. Such optical element is classified as homogeneous [4].

Let us eventually consider the example of an incident light beam with any coherency matrix $\mathbf{H}_{1}$ and an emerging light with any coherency matrix $\mathbf{H}_{2}, \mathbf{J}$ may have nonorthogonal eigenpolarizations and may be associated to an inhomogeneous optical elements.

\section{4 - Conclusion}

In this work, the question coherency matrix propagation of a light beam is addressed by means of the analysis of interpolation processes between two physical situations. The basic point is that the state of light beam or the path in a medium to go from a physical situation at distance $z_{1}$ to another one at distance $z_{2}$ should be related to the correlation between both these physical situations. Solving the problem in the same way for light beam and medium is enable using coherency matrices formalism. The physical situations are defined according to the second order statistical properties of the underlying process. Equivalence classes are derived from the definition of a group action on the set of coherency matrices. The geodesic curves on each equivalence class define the process of interpolation. The general solution is derived as a symbolic equation and the solution is explicitly developed for the particular situation of uncorrelated statistical processes. Under this hypothesis, the physical significance of the interpolation technique based on the affine invariant distance is obvious. Interpolating coherency matrix describes the propagation of a light beam into a uniform non depolarizing medium characterized by a differential Jones matrix completely determined by the far points of the interpolation curve up to a unitary matrix. Other explicit developments needing further studies of the geodesic curves using local coordinates are under investigation. 


\section{Appendix A}

The Riemannian metric we used, was originally studied for the space of multivariate normal distribution by Rao. A unified approach to the derivation of metrics in the spaces of probability density function was proposed by Burbea and Rao [12].

In the context of this article, the square of the distance between to infinitesimally close elements $\mathbf{H}$ and $\mathbf{H}+\mathrm{d} \mathbf{H}$ of $H D P(4)$ is defined by:

$$
\|\mathrm{d} \mathbf{H}\|_{\mathbf{H}}^{2}=\frac{1}{2} \operatorname{tr}\left[\left(\mathbf{H}^{-1} \mathrm{~d} \mathbf{H}\right)^{2}\right]
$$

where $\operatorname{tr}(\mathbf{A})$ stands for the trace of $\mathbf{A}$.

Thus for any elements $\mathbf{A}, \mathbf{B}$ and $\mathbf{C}$ of $H D P(4)$, the inner product of $\mathbf{A}$ and $\mathbf{B}$ relative to $\mathbf{C}$ is defined by:

$$
\langle\mathbf{A}, \mathbf{B}\rangle_{\mathbf{C}}=\frac{1}{2} \operatorname{tr}\left[\mathbf{C}^{-1} \mathbf{A} \mathbf{C}^{-1} \mathbf{B}\right]
$$

\section{Appendix B}

We consider the curve defined by:

$$
\mathbf{H}(z)=\exp (z \mathbf{N}) \mathbf{H}_{1}[\exp (z \mathbf{N})]^{\dagger}
$$

with $\mathbf{N}$ defined by

$$
\mathbf{N}=\frac{1}{2} \mathbf{H}_{1}^{1 / 2}\left[\log \left[\mathbf{H}_{1}^{-1 / 2} \mathbf{H}_{2}\left(\mathbf{H}_{1}^{-1 / 2}\right)^{\dagger}\right]+\mathbf{F}\right]\left(\mathbf{H}_{1}^{-1 / 2}\right)^{\dagger}
$$

where $\mathbf{H}_{1}$ and $\mathbf{H}_{2}$ are hermitian matrices and $\mathbf{F}$ is a skew-hermitian matrix commuting with

$$
\log \left[\mathbf{H}_{1}^{-1 / 2} \mathbf{H}_{2}\left(\mathbf{H}_{1}^{-1 / 2}\right)^{\dagger}\right]
$$

According to the classical properties of matrix exponential:

$$
\begin{aligned}
& \exp (z \mathbf{N})=\mathbf{H}_{1}^{1 / 2} \exp \left[\frac{z}{2}\left[\log \left[\mathbf{H}_{1}^{-1 / 2} \mathbf{H}_{2}\left(\mathbf{H}_{1}^{-1 / 2}\right)^{\dagger}\right]+\mathbf{F}\right]\right]\left(\mathbf{H}_{1}^{-1 / 2}\right) \\
& \exp (z \mathbf{N})=\mathbf{H}_{1}^{1 / 2} \exp \left[\frac{z}{2} \log \left[\mathbf{H}_{1}^{-1 / 2} \mathbf{H}_{2}\left(\mathbf{H}_{1}^{-1 / 2}\right)^{\dagger}\right]\right] \cdot \exp \left[\frac{z}{2} \mathbf{F}\right]\left(\mathbf{H}_{1}^{-1 / 2}\right)
\end{aligned}
$$

Thus:

$$
\mathbf{H}(z)=\mathbf{H}_{1}^{1 / 2} \exp \left[\frac{z}{2} \log \left[\mathbf{H}_{1}^{-1 / 2} \mathbf{H}_{2}\left(\mathbf{H}_{1}^{-1 / 2}\right)^{\dagger}\right]\right] \exp \left[\frac{z}{2} \mathbf{F}\right] \exp \left[\frac{z}{2} \mathbf{F}^{\dagger}\right] \exp \left[\frac{z}{2} \log \left[\mathbf{H}_{1}^{-1 / 2} \mathbf{H}_{2}\left(\mathbf{H}_{1}^{-1 / 2}\right)^{\dagger}\right]\right]\left(\mathbf{H}_{1}^{1 / 2}\right)^{\dagger}
$$

Since $\mathbf{F}^{\dagger}=-\mathbf{F}$, we have:

$$
\mathbf{H}(z)=\mathbf{H}_{1}^{1 / 2} \exp \left[z \log \left[\mathbf{H}_{1}^{-1 / 2} \mathbf{H}_{2}\left(\mathbf{H}_{1}^{-1 / 2}\right)^{\dagger}\right]\right]\left(\mathbf{H}_{1}^{1 / 2}\right)^{\dagger}
$$

So $\mathbf{H}(z)=\mathbf{H}_{(H 1, \boldsymbol{W})}(z)$. 


\section{References}

1. R. C. Jones, "A new calculus for the treatment of optical systems," J. Opt. Soc. Am. 38, 671-685 (1948)

2. R. M. A. Azzam, "Propagation of partially polarized light through anisotropic media with or without depolarization: A differential 4x4 matrix calculus," J. Opt. Soc. Am. 68, 1756-1767 (1978)

3. R. Barakat, "Exponential versions of the Jones and Mueller-Jones polarization matrices," J. Opt. Soc. Am. A 13, 158-163 (1996)

4. Cristian Brosseau, Fondamentals of polarized light: a statistical optics approach, (John Wiley, New York, 1998)

5. J.W. Goodman, Statistical Optics, (John Winley \& Son, New-York, 1985)

6. P. Réfrégier, "Symmetries in coherence theory of partially polarized light," J. Math. Phys. 48, 033303 1-14 (2007)

7. Philippe Réfrégier, "Mutual information-based degrees of coherence of partially polarized light with Gaussian fluctuations," Opt. Lett. 30, 3117-3119 (2005)

8. Williams M. Boothby, An introduction to Differentiable Manifolds and Riemannian GeometrySecond Edition, (Academic Press, Inc., Orlando, 1986)

9. V. Devlaminck and P. Terrier, "Geodesic distance on non-singular coherency matrix space in polarization optics," J. Opt. Soc. Am. A 27, 1756-1763 (2010)

10. Sigudur Helgason, Differential Geometry and Symmetric spaces, (Academic Press, New York, 1962)

11. X. Pennec, P. Fillard, N. Ayache, "A Riemannian Framework for Tensor Computing," Int. J. Comput. Vis. 66, 41-66 (2006)

12. J. Burbea and C. Rao, "Entropy differential metric, distance and divergence measures in probability spaces: A unified approach," J. Multivariate Analysis 12, 575-596 (1982) 